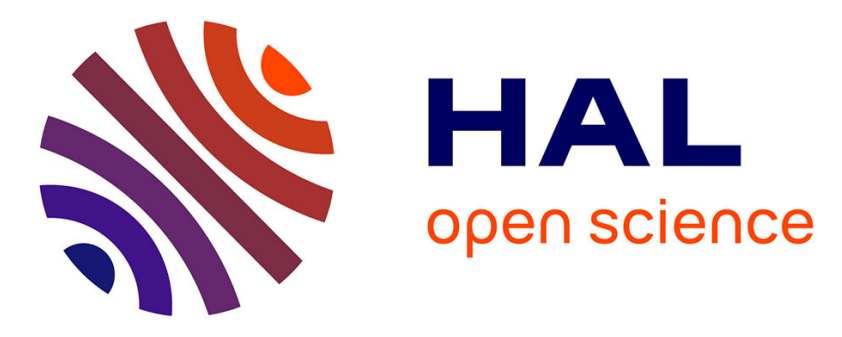

\title{
Study on Optimal Design Based on Direct Coupling Between a FEM Simulation Model and L-BFGS-B Algorithm
}

Mohamed Saïd Berkani, Stefan Giurgea, Christophe Espanet, Jean Coulomb, Christophe Kieffer

\section{To cite this version:}

Mohamed Saïd Berkani, Stefan Giurgea, Christophe Espanet, Jean Coulomb, Christophe Kieffer. Study on Optimal Design Based on Direct Coupling Between a FEM Simulation Model and L-BFGS-B Algorithm. IEEE Transactions on Magnetics, 2013, 49 (5), pp.2149-2152. 10.1109/TMAG.2013.2245871 . hal-02300100

\section{HAL Id: hal-02300100 https://hal.science/hal-02300100}

Submitted on 6 Oct 2021

HAL is a multi-disciplinary open access archive for the deposit and dissemination of scientific research documents, whether they are published or not. The documents may come from teaching and research institutions in France or abroad, or from public or private research centers.
L'archive ouverte pluridisciplinaire HAL, est destinée au dépôt et à la diffusion de documents scientifiques de niveau recherche, publiés ou non, émanant des établissements d'enseignement et de recherche français ou étrangers, des laboratoires publics ou privés. 


\title{
Study on Optimal Design Based on Direct Coupling Between a FEM Simulation Model and L-BFGS-B Algorithm
}

\author{
M. S. Berkani ${ }^{1}$, S. Giurgea ${ }^{1}$, C. Espanet ${ }^{1}$, J. L. Coulomb ${ }^{2}$, and C. Kieffer ${ }^{1}$ \\ ${ }^{1}$ ENERGIE Department, FEMTO-ST Institute, F-90010 Belfort, France \\ ${ }^{2}$ G2Elab, BP-38402 Grenoble, France
}

\begin{abstract}
This paper investigates the capability of a Quasi-Newton optimization algorithm, the L-BFGS-B one, to reduce the drawbacks of the direct coupling with FEM models. After a short description of the L-BFGS-B algorithm, the authors have tested it to the TEAM Workshop Problem 25. L-BFGS-B algorithm issues linked to FEM estimation of the objective function have been analyzed. Finally the authors present the results of an electromagnetic device optimal design, using a large number of parameters (47).
\end{abstract}

Index Terms-Finite element methods, optimization methods.

\section{INTRODUCTION}

I $\mathrm{N}$ the context of a growing part of electrical power in the industry, there is a strong need to develop fast and efficient design methodologies. In this situation, a numerical model based on the finite element method (FEM), used in the optimization process, provides both the benefits of a fast development and of the accuracy. On the other hand, several drawbacks have been identified in the past few decades; two of them seem to be the most important. First, the FEM model could require an important computation time to estimate the objective function, which doesn't enable to control the global computation cost of the optimization. A second drawback is linked to the objective function that may be noisy due to the effects of mesh variation [1]. For example, Fig. 1 shows a zoom on the variation of the objective function estimated by the FEM model of the TEAM Workshop Problem 25 (TEAM 25) [2], for the parameter $\mathrm{L}_{2}$ variation. In this example the others parameters are fixed at their initial value (see Table I). This noise could perturb the gradient-based algorithms. One way to reduce computation time is to substitute the objective function, estimated by the FEM, with an approximation model. This strategy requires less computation time, since it reduces the number of FEM simulations. Different approximation methods have been proposed such as polynomial regression [3], radial basis function [4], multiquadratic basis functions [5], and kriging [6]. These solutions provide a continuous and smooth approximation and are compatible with deterministic and stochastic optimization algorithms. Nevertheless, to calibrate an approximation model, several points are needed on each parameter that implies a number of FEM estimations, which depends exponentially on the parameters number. This negative feature can be avoided by choosing in the approximation model only the most significant parameters of the FEM model [7]. Approximation errors may also influence the precision of the final result.

The purpose of this contribution is to investigate the capability of a Quasi-Newton based algorithm, the L-BFGS-B one, to produce a fine result by using direct coupling with an interfaced FEM model. To achieve this study, we consider the

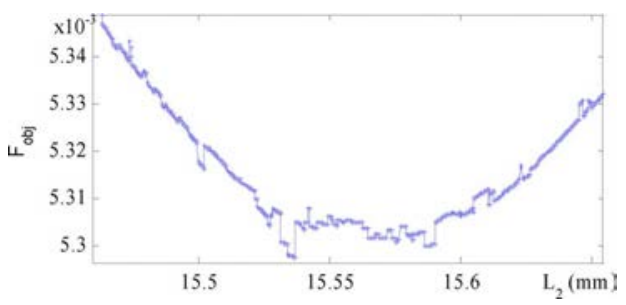

Fig. 1. Objective function noise observed on FEM simulation of TEAM Workshop Problem 25.

TABLE I

CONSIDERED GEOMETRIC PARAMETERS

\begin{tabular}{llll}
\hline Symbol & $\begin{array}{l}\text { Feasibility } \\
\text { Domain }\end{array}$ & Initial Value & Optimal Value \\
\hline $\mathrm{R}_{1}$ & {$[5.0 \div 9.4] \mathrm{mm}$} & $7.2 \mathrm{~mm}$ & 7.347 \\
$\mathrm{~L}_{2}$ & {$[12.6 \div 18.0] \mathrm{mm}$} & $15.3 \mathrm{~mm}$ & 14.527 \\
$\mathrm{~L}_{3}$ & {$[14.0 \div 45.0] \mathrm{mm}$} & $29.5 \mathrm{~mm}$ & 14.243 \\
$\mathrm{~L}_{4}$ & {$[4.0 \div 19.0] \mathrm{mm}$} & $11.5 \mathrm{~mm}$ & 14.357
\end{tabular}

TEAM 25 problem. In the Section II, the authors describe the L-FBGS-B algorithm. Then, in the Section III, they present the optimisation problem formulation of TEAM 25. In the Section IV, they analyse the particularities of L-BFGS-B algorithm coupled to a FEM model through the use case TEAM 25. Finally, in Section $\mathrm{V}$, the authors present the results of an electromagnetic device optimal design; the interest of this example is that the optimization uses 47 parameters, which is quite large for a FEM model.

\section{L-BFGS-B OVERVIEW}

As described in [8] the L-BFGS-B algorithm is a QuasiNewton based algorithm that allows finding the minimum of an $\mathrm{n}$ dimensional function:

$$
\mathbf{x}^{*}=\underset{\mathbf{x} \in \bar{D}}{\operatorname{Arg} \min }\{f(\mathbf{x})\}
$$

The feasibility domain $\bar{D}$ accepts simple bounds on variables and can be defined by a hyper cube with minimal and maximal limit of each parameter as shown in the following:

$$
\bar{D}=\left\{\mathbf{x} \in R^{n} \mid l_{i} \leq x_{i} \leq h_{i}, \forall i \in 1 \ldots n\right\}
$$

At each iteration, the last values of the function, $f_{k}$, and of its gradient, $g_{k}^{T}$, are computed; the hessian matrix approximation 


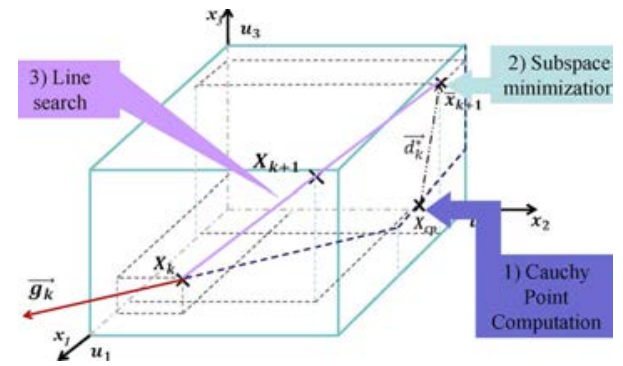

Fig. 2. L-BFGS-B algorithm overview.

$B_{k}$ is updated. Quadratic approximation of the objective function is then used at different steps of the algorithm:

$$
m_{k}(\mathbf{x})=f_{k}+\mathbf{g}_{k}^{T}\left(\mathbf{x}-\mathbf{x}_{k}\right)+\frac{1}{2}\left(\mathbf{x}-\mathbf{x}_{k}\right)^{T} \mathbf{B}_{k}\left(\mathbf{x}-\mathbf{x}_{k}\right)
$$

The updating mechanism of $B_{k}$ is realized by using the last $p$ correction pairs $\left.\left\{s_{i}, y_{i}\right\}\right|_{i=k-1, \ldots k-p}$ defined by:

$$
\mathrm{s}_{\mathrm{k}}=\mathrm{x}_{\mathrm{k}+1}-\mathrm{x}_{\mathrm{k}} ; \quad \mathrm{y}_{\mathrm{k}}=\mathrm{g}_{\mathrm{k}+1}-\mathrm{g}_{\mathrm{k}},
$$

representing the evolutions of the current point and of the objective function gradient between two successive iterations. This is one aspect that can explain the behavior of the algorithm coupled with FEM model. Three stages compose an iteration of the L-BFGS-B algorithm (Fig. 2):

First Step-Cauchy Point Computation: The objective of this step is to find the local minimum $\mathbf{x}^{c}$ of quadratic approximation of the objective function, starting from the current point $x_{k}$, on the path defined by the projection of the steepest descent direction on the feasible domain. On this projection path, a point $\mathrm{x}$ can be expressed by its travel distance $t$ from the starting point $x_{k}$. Traveling on the opposite gradient direction, one by one, the $\mathrm{n}$ variables of objective function will reach those limits at $n$ values of $t:\left\{t^{0}, \ldots t^{j-1}, t^{j}, \ldots t^{n-1}\right\}$ For $t \in\left[t^{j-1}, t^{j}\right]$ the corresponding point $\mathrm{x}$ can be expressed:

$$
\mathbf{x}(t)=\mathbf{x}\left(t^{j-1}\right)+\Delta t d^{j-1}
$$

with $\Delta t=t-t^{j-1}$, and $d^{j-1}$, the steepest descent direction, projected on the feasibility domain.

On the segment $\left[t^{j-1}, t^{j}\right]$, the quadratic approximation $m(\mathbf{x})$ can be expressed as a one-dimensional quadratic expression in $\Delta t$ :

$$
\hat{m}(\Delta t)=f_{j-1}+f_{j-1}^{\prime} \Delta t+\frac{1}{2} f_{j-1}^{\prime \prime} \Delta t^{2}
$$

where the scalar values $f_{j-1}, f_{j-1}^{\prime}, f_{j-1}^{\prime \prime}$ are obtained using the values of $f_{k}, g_{k}^{T}$ and $B_{k}$. The optimal $\Delta t^{*}$ value can be estimated:

$\Delta t^{*}=-\left(f_{j-1}^{\prime} / f_{j-1}^{\prime \prime}\right)$. Its corresponding point represents the Cauchy point:

$$
\mathbf{x}^{c}=\mathbf{x}\left(t^{j-1}+\Delta t^{*}\right)=\mathbf{x}\left(t^{j-1}\right)+\Delta t^{*} d^{j-1} .
$$

The indexes of the variables fixed on their limits, during the Cauchy point computation, are grouped in a set $A\left(\mathbf{x}^{\mathbf{C}}\right)$.

Second Step-Subspace Minimization: It represents a Quasi Newton stage starting from the Cauchy Point obtained previously. A new descent direction is obtained by searching the

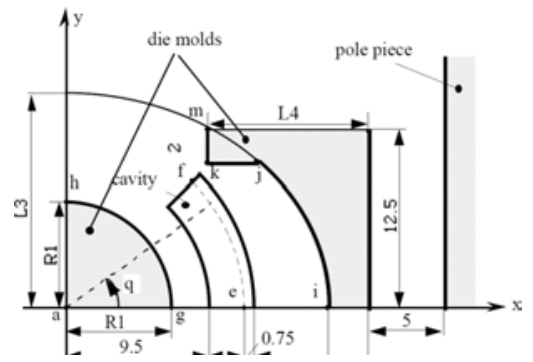

Fig. 3. TEAM Workshop Problem 25 geometry.

minimum of (3) on the free variable subspace, by using the following formulation:

$$
\overline{\mathbf{x}}_{k+1}=\underset{\mathbf{x} \in \bar{D}_{S}}{\operatorname{Arg} \min }\left\{m_{k}(\mathbf{x})\right\}
$$

In this step, the feasibility domain is reduced at a subspace of the feasibility domain $\bar{D}$, by considering as free variables, the variables that are not fixed on limits during the Cauchy point step:

$$
\bar{D}_{S}=\left\{\mathbf{x} \in R^{N} \mid l_{i} \leq x_{i} \leq h_{i}, \forall i \notin A\left(x^{C}\right)\right\}
$$

The others $(n-t)$ variables are fixed on their boundary value obtained during the Cauchy point step.

$$
\mathbf{x}[i]=\mathbf{x}^{C}[i], \quad \forall i \in A\left(\mathbf{x}^{C}\right)
$$

The Third Step - Line Search: It achieves a linear search on $\left(\mathbf{x}_{k}, \overline{\mathbf{x}}_{k+1}\right)$ to find a new point $\mathbf{x}_{k+1}$ that satisfies a sufficient decrease condition and a curvature condition (known also as Wolfe's conditions). The line search implies successive one-dimensional interpolations (quadratic or cubic) to compute an acceptable step from $\mathbf{x}_{k}$ point to $\overline{\mathbf{x}}_{k+1}$ point. This stage requires additional estimations of the function $f$ and of its gradient, $g^{T}$, if $\overline{\mathbf{x}}_{k+1}$ doesn't satisfy the Wolfe conditions.

\section{APPLICATION}

The algorithm has been tested with using the classical optimization problem TEAM 25 [2]. The goal is to optimize the geometry of a die mold used in the production of permanent magnets. The die mold geometry is described by 4 parameters: R1, L2, L3 and L4 as shown in Fig. 3 [2]. We have to find the optimal value of the four geometrical parameters, to obtain a radial magnetic field on the arc ef. Reference value of the flux density presents a sinusoidal distribution of $\mathrm{B}_{\mathrm{x}}$ and $\mathrm{B}_{\mathrm{y}}$ components:

$$
\left\{\begin{array}{l}
B_{x 0}=0.35 \cdot \cos \theta \\
B_{y 0}=0.35 \cdot \sin \theta
\end{array} .\right.
$$

This objective function is obtained by minimizing the residual sum of squares between the reference values and FEM simulated values, on ten different points on the arc ef:

$$
F_{\text {obj }}=\sum_{i=1}^{10}\left\{\left(B_{x_{-} i}-B_{x 0_{-} i}\right)^{2}+\left(B_{y_{-} i}-B_{y 0_{-} i}\right)^{2}\right\}
$$

The objective function is strictly positive. Sufficiently small value of the objective function can be chosen as STOP criteria for this problem. The definition domain of each parameter has been normalized into the interval $[0,1]$. In the gradient evaluation, partial derivatives are obtained using the Newton's difference quotient. The same normalized variation $\varepsilon$ has been considered for all normalized parameters. Five FEM computations 
TABLE II

NUMBER OF FEM ESTIMATIONS AND OPTIMIZATION RESULTS

\begin{tabular}{|l|l|l|l|l|l|l|l}
\hline & $\varepsilon$ & $p$ & $\mathrm{n}_{f, g}$ & $\mathrm{n}_{\text {fem }}$ & $\mathrm{F}_{\text {obj }}$ & $\begin{array}{l}\text { Final } \\
\mathrm{n}_{\text {fem }}\end{array}$ & $\begin{array}{l}\text { Final } \\
\mathrm{F}_{\text {obj }}\end{array}$ \\
\hline lbfgsb & 0.004 & 9 & 50 & 250 & $3.60 \mathrm{E}-4$ & 1025 & $4.67 \mathrm{E}-5$ \\
\hline lbfgsb & 0.004 & 30 & 37 & 175 & $4.36 \mathrm{E}-4$ & 815 & $4.60 \mathrm{E}-5$ \\
\hline Ibfgsb & 0.004 & 20 & 37 & 175 & $4.36 \mathrm{E}-4$ & 815 & $4.60 \mathrm{E}-5$ \\
\hline lbfgsb & 0.008 & 9 & 39 & 195 & $3.94 \mathrm{E}-4$ & 540 & $8.25 \mathrm{E}-5$ \\
\hline Ibfgsb & 0.008 & 30 & 27 & 135 & $1.89 \mathrm{E}-4$ & 260 & $1.64 \mathrm{E}-4$ \\
\hline lbfgsb & 0.012 & 9 & - & - & - & 440 & $3.59 \mathrm{E}-3$ \\
\hline Ibfgsb & 0.012 & 30 & 70 & 350 & $4.13 \mathrm{E}-4$ & 1125 & $1.12 \mathrm{E}-4$ \\
\hline lbfgsb & 0.05 & 30, & - & - & - & 250 & $5.49 \mathrm{E}-3$ \\
\hline rs1[2] & - & - & - & 343 & $6.29 \mathrm{E}-4$ & - & - \\
\hline rs2[2] & - & - & - & 95 & $4.73 \mathrm{E}-4$ & - & - \\
\hline
\end{tabular}

are used to compute $\{f, g\}$, for a pointx $=\left[R_{1}, L_{2}, L_{3}, L_{4}\right]$. The optimization parameters, their initial values and feasibility domains are given in Table I.

\section{Results AND AlgORIthm ANALysis}

In this section, some important issues of coupling L-BFGS-B algorithm with a FEM model are analyzed, such as the compatibility with the FEM model and the influence of $\varepsilon$ and $p$ parameters. We analyze also two observed difficulties of this methodology due to the Line Search stage and STOP criteria of the algorithm.

\section{A. Compatibility of the Algorithm With a FEM Model}

Some previous work shows the capability of response surfaces to deal with the objective function presenting a mesh noise. On the same optimization problem a lower number of iteration has been obtained in [7] after a screening stage, by an adapting response surface applied on 3 parameters. The results obtained in [7] are resumed in Table II, (rs1 and rs2 results).

Using Quasi-Newton research directions, L-BFGS-B implicitly uses quadratic approximations that may have the same behaviour of a response surface, in the case of a noisy objective function. For each iteration, the algorithm operates two implicit local approximations. When using a direct connection with the FEM solver, we can anticipate that this mechanism blur the discontinuities due to the mesh variation. The method can take into consideration an important number of parameters. This enables a strong reduction of the objective function, when an important number of parameters are influent. As it is shown in the Table II, the minimum value of the objective function obtained for $\varepsilon=0.004$ and $p=30$, with 815 FEM estimations was $\mathrm{F}_{\mathrm{obj}}=4.6 \mathrm{E}-5$ which divides by ten the previous results obtained in [7] (rs2 in Table II). The results of this optimization are given in Table I. Computing time dedicated to the algorithm computation was $\mathrm{t} 1=0.6(\mathrm{~s})$ and the one dedicated to FEM estimation was $8532(\mathrm{~s})$.

\section{B. Influence of $\varepsilon$ (The Gradient Computation Normalized Variation)}

The influence of the normalized variation used in the gradient computation, $\varepsilon$, has been tested by considering 4 values. Fig. 4 shows the evolution of the objective function depending on the number of estimations of $\{f, g\}$ for the same number of correction pairs $p=30$. It can be observed that $\varepsilon$ is influent on the

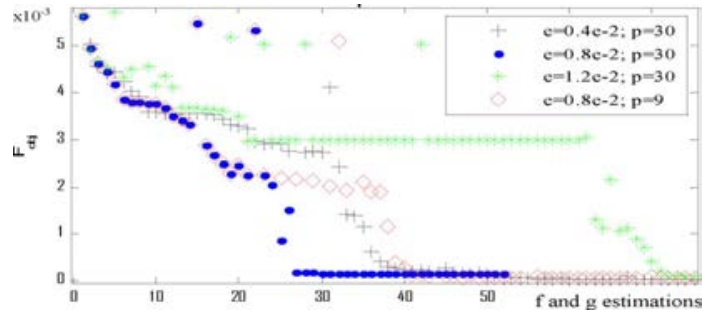

Fig. 4. Evolution of the objective function for different $p, \varepsilon(e)$.

gradient accuracy and therefore on the speed of convergence. This dependence is not linear and the better performance has been obtained for $\varepsilon=0.008$.

\section{Influence of the Number of the Last p Correction Pairs Considered in Hessian Approximation}

The parameter $p$ influences the convergence too. The considered example shows a better convergence for a greater value of $p$. In Fig. 4 we can observe also the evolution of the objective function when the algorithm use $\varepsilon=0.008$ for $p=9$ and $p=30$ values.

Expression (4) shows that a number p of previous results, estimated on the FEM model, are used to actualize the Hessian approximation. Acting on the number of the last $p$ experiments will provide a local behavior of the quadratic approximation (for a small value of $p$ ) or global one (for a higher value of $p$ ). This parameter has also an influence on the approximation of the Hessian matrix, B. As shown in [8], a lower value of $p$ limits the memory and the time computation necessary to estimate the approximation of the Hessian matrix, used in the Quasi-Newton process. However, the algorithm computation time is negligible in comparison with the FEM computation time. Table II synthesizes the results obtained for different configurations $\varepsilon$ and $p$ of the algorithm. In order to have a comparison between the different $\{p, \varepsilon\}$ configurations, and also to compare the results obtained from L-BFGS-B with other results from literature, columns $\mathrm{n}_{\mathrm{f}, \mathrm{g}}, \mathrm{n}_{\text {fem }}$ and $\mathrm{F}_{\mathrm{obj}}$ from Table II shows the necessary number of iterations to obtain FEM computed value of the objective function $\mathrm{F}_{\mathrm{obj}} \leq 4.6 \mathrm{E}-4$. Last columns "Final $\mathrm{n}_{\mathrm{fem}}$ " and "Final $\mathrm{F}_{\mathrm{obj}}$ " represent the result obtained when the optimization process is stopped because the line search process doesn't succeed.

\section{Analysis on Line Search Stage}

During the line search step, the objective functions and its gradient are calculated successively to find a point $\mathbf{x}_{k+1}$ that satisfies the Wolfe conditions. As in the line search, $f$ and $g$ are directly estimated on the Objective Function (FEM estimated), this step can correct the approximation errors linked to the quadratic approximation. Fig. 5(a) presents the L-BFGS-B evolution for $\varepsilon=0.008$ and $p=30$. We observe also the number of line search per iteration. The minimum point (presented in Table II) has been reached after $27\{f, g\}$ estimations and 19 iterations. A large number of iterations execute only one estimation $\{f, g\}$ for the line search step. This happens when $\overline{\mathbf{x}}_{k+1}$ satisfies both Wolfe conditions. For a positive definite $B_{k}$, [8] has been proven that that the approximation solution $\overline{\mathbf{x}}_{k+1}$ of the quadratic problem defined in the subspace minimization step defines a descent direction $d_{k}=\overline{\mathbf{x}}_{\mathbf{k}+\mathbf{1}}-\mathbf{x}_{\mathbf{k}}$ for 


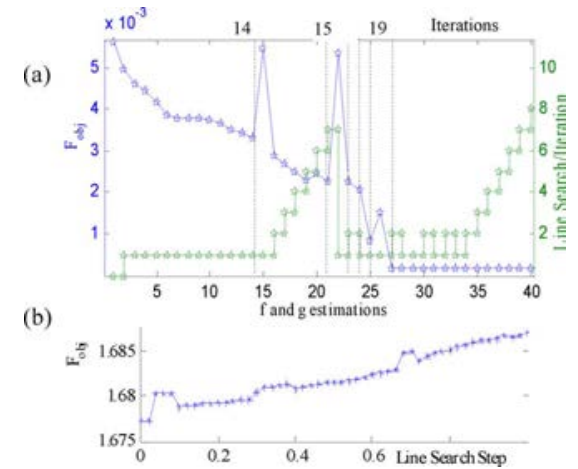

Fig. 5. (a) Number of line search per iteration, (b) Objective function evolution on the last line search.

the objective function. One problem in coupling LBFGS algorithm with a FEM model corresponds to the situation where the line search doesn't find $\mathbf{x}_{\mathbf{k}+\mathbf{1}} \in\left(\mathbf{x}_{\mathbf{k}}, \overline{\mathbf{x}}_{\mathbf{k}+\mathbf{1}}\right)$ satisfying Wolfe conditions. This situation occurs when the objective function is close to the theoretical optimum point. In this situation, the algorithm stops before reaching a convergence criterion. Fig. 5(b) shows the objective function estimation on the last line search segment $\left(\mathbf{x}_{\mathbf{k}}, \overline{\mathbf{x}}_{\mathbf{k}+\mathbf{1}}\right)$ before the algorithm is stopped, when the line search process doesn't succeed, for $\varepsilon=0.008$ and $p=30$. The real objective function does not descend in this last interval.

One explanation could be the noise of the gradient which is more significant near the optimal point. Even if the quadratic approximation function (3) is descending, the real function may increase within the small interval of the line search interval. In Table II, "Final $\mathrm{n}_{\mathrm{fem}}$ " and "Final $\mathrm{F}_{\mathrm{obj}}$ " results were obtained by this criterion and it represents the last iteration that corresponds to a decrease of the objective function.

\section{LARge Number of Parameters Optimization: Magneto Caloric Device Optimization}

To illustrate the behavior of L-BFGS-B coupled to a practical FEM model having a large number of parameters, we present here the case of a thermo magnetic device design presented in [9]. The sizing of the rotor magnets (Fig. 6(a)) is carried out in order to obtain a maximum flux density variation $\Delta \mathrm{B}$ in the regenerator air gap, to ensure the performance of the magneto caloric device. To limit the saturation, the extremity of the rotor pole is realized on a cobalt-iron alloy "Vacoflux" with a high saturation flux density [10]. An initial optimal design has been obtained with using only two parameters. The objective function to minimize is the quadratic error sum between the reference value of the flux density module $\left(\mathrm{B}_{\mathrm{ref}}\right)$ and the value of the FEM estimated value $\left(\mathrm{B}_{2 \mathrm{p}}\right)$, for two test zones $\mathrm{B}_{\text {ref1 }}=0(\mathrm{~T})$ in $\Gamma_{1}=\left(0^{\circ}, 9^{\circ}\right)$ and $\mathrm{B}_{\mathrm{ref} 2}=2.2(\mathrm{~T})$ in $\Gamma_{2}=\left(36^{\circ}, 45^{\circ}\right)$. The solution has been obtained by a direct coupling of the FEM model and L-BFGS-B algorithm. This optimal geometry and the flux distribution are shown in Fig. 6(b). The reference value and the FEM computed value are presented in Fig. 6(d). To improve the result, the problem has been modified, by increasing the number of parameters of the FEM model from 2 to 47 parameters characterizing the magnets geometry and their orientation. Then, the L-BFGS-B was coupled to this model. The results were obtained after $72 \mathrm{~h}$ of computation and about 7000 FEM estimations. The resulting geometry and the magnets orientation
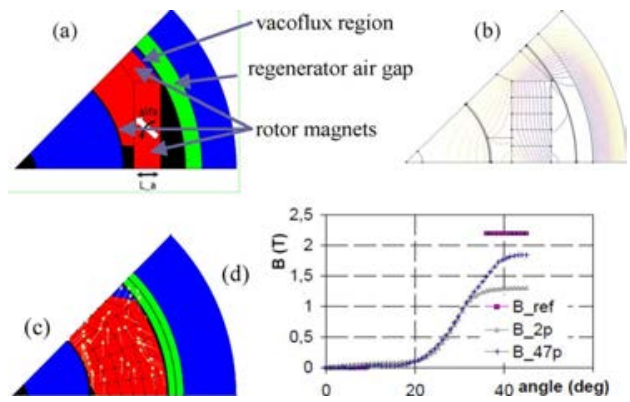

Fig. 6. (a) Two parameters design of the initial configuration, (b) Magnetic flux distribution, (c) Optimal 47 parameters design, (d) Flux density in the regenerator: reference and computed values.

are shown in Fig. 6(c). We can observe in Fig. 6(d). a strong improvement of the device: an average value for $\Delta \mathrm{B}$ between the two test zones $\left\langle\Delta \mathrm{B}_{47 \mathrm{p}}\right\rangle=1.74(\mathrm{~T})$ is reached in comparison with $\left\langle\Delta \mathrm{B}_{2 \mathrm{p}}\right\rangle=1.29(\mathrm{~T})$ obtained with the initial optimal design.

\section{CONCLUSION}

The analysis of the L-BFGS-B algorithm and the numerical experimentation proved the capability of this algorithm to produce an accurate result when is directly coupled with an interfaced FEM model. The influence of two parameters has been observed on the algorithm convergence: the gradient computation normalized variation and the last $\mathrm{p}$ correction pairs considered in Hessian approximation. A study of the line search stage shows a different behavior when the objective function is FEM estimated. Finally the algorithm capability to solve a practical OP with a large number of parameters (47) has been confirmed on the FEM model of special device for magnetocaloric refrigeration.

\section{REFERENCES}

[1] S. Ratnajeevan, H. Hoole, K. Weeber, and S. Subramaniam, "Fictitious minima of object functions, finiteelement meshes, and edge elements in electromagnetic device synthesis," IEEE Trans. Magn., vol. 27, pp. 5214-5216, Nov. 1991.

[2] N. Takahashi, K. Ebihara, K. Yoshida, T. Nakata, K. Ohashi, and K. Miyata, "Investigation of simulated annealing method and its application to optimal design of die mold for orientation of magnetic powder," IEEE Trans. Magn., vol. 32, pp. 1210-1213, 1996

[3] S.-I. Kim1, J.-P. Hong1, Y.-K. Kim2, H. Nam3, and H.-I. Choi, "Optimal design of slotless-type PMLSM considering multiple responses by response surface methodology," IEEE Trans. Magn., vol. 42, no. 4, pp. 1219-1223, Apr. 2006.

[4] T. Ishikawa, Y. Tsukui, and M. Matsunami, "A combined method for the global optimization using radial basis function and deterministic approach," IEEE Trans. Magn., vol. 35, no. 3, pp. 1730-1733, 1999.

[5] P. Alotto, A. Caiti, G. Molinari, and M. Repetto, "A multiquadrics based algorithm for the acceleration of simulated annealing optimization procedures," IEEE Trans. Magn., vol. 32, no. 3, pp. 1198-1201, May 1996.

[6] L. Lebensztajn, C. A. R. Marretto, M. C. Costa, and J. L. Coulomb, "Kriging: A useful tool for electromagnetic device optimization," IEEE Trans. Magn., vol. 40, no. 2, pp. 1196-1199, 2004.

[7] M. C. Costa, J. L. Coulomb, Y. Maréchal, and S. I. Nabeta, "An adaptive method applied to the diffuse element approximation in optimization process," IEEE Trans. Magn., vol. 37, no. 5, pp. 3418-3422, Sept. 2001.

[8] R. H. Byrd, P. Lu, J. Nocedal, and C. Zhu, "A limited memory algorithm for bound constrained optimization," SIAM J. Sci. Comput., vol. 16, no. 5, pp. 1190-1208, Sept. 1995.

[9] C. Kieffer, F. Gustin, S. Giurgea, C. Espanet, and M. Roze, "Design of an electrical machine for an integrated magnetocaloric coupling system," in Int. Conf. Magnetic Refrigeration at Room Temperature, Thermag V, Grenoble, France, Sep. 17-20, 2012.

[10] M. Centner and R. Hanitsch, in U. Schafer Comparison of High-Speed Induction Motors Employing Cobalt-Iron and Silicon Electrical Steel ICEM-2008 Conf., Sep. 2008, paper ID 1386. 